Please do not remove this page

RMIT

UNIVERSITY

\title{
Application of \\ Nonlinear-Autoregressive-Exogenous model to predict the hysteretic behaviour of passive control systems
}

Chan, Ricky; Yuen, Jason; Lee, Eric; Arashpour, Mehrdad

https://researchrepository.rmit.edu.au/esploro/outputs/9921862149501341/filesAndLinks?institution=61RMIT_INST\&index=null

Chan, R., Yuen, J., Lee, E., \& Arashpour, M. (2015). Application of Nonlinear-Autoregressive-Exogenous model to predict the hysteretic behaviour of passive control systems. Engineering Structures, 85, 1-10. https://doi.org/10.1016/j.engstruct.2014.12.007

Document Version: Accepted Manuscript

Published Version: https://doi.org/10.1016/j.engstruct.2014.12.007

Repository homepage: https://researchrepository.rmit.edu.au

(c) 2014 Elsevier Ltd. All rights reserved.

Downloaded On 2023/04/26 17:52:31 +1000 
Thank you for downloading this document from the RMIT Research Repository.

The RMIT Research Repository is an open access database showcasing the research outputs of RMIT University researchers.

RMIT Research Repository: http://researchbank.rmit.edu.aul

\section{Citation:}

Chan, R, Yuen, J, Lee, E and Arashpour, M 2015, 'Application of

Nonlinear-Autoregressive-Exogenous model to predict the hysteretic behaviour of passive control systems', Engineering Structures, vol. 85, pp. 1-10.

See this record in the RMIT Research Repository at:

https://researchbank.rmit.edu.au/view/rmit:29792

Version: Accepted Manuscript

\section{Copyright Statement:}

(c) 2014 Elsevier Ltd. All rights reserved. () 2014. This manuscript version is made available under the CC-BY-NC-ND 4.0 license http://creativecommons.org/ licenses/by-nc-nd/4.0/

Link to Published Version:

http://dx.doi.org/10.1016/j.engstruct.2014.12.007 


\title{
Application of Nonlinear-Autoregressive-Exogenous model to predict the hysteretic behaviour of passive control systems
}

\author{
Ricky W.K. Chan ${ }^{1}$, Jason K.K. Yuen ${ }^{2}$, Eric W.M. Lee ${ }^{2 *}$, and Mehrdad Arashpour ${ }^{3}$ \\ ${ }^{1}$ School of Civil, Environmental and Chemical Engineering, RMIT University, Melbourne, \\ Australia \\ ${ }^{2}$ Department of Civil and Architectural Engineering, City University of Hong Kong, Kowloon Tong, \\ Hong Kong, China \\ ${ }^{3}$ School of Property, Construction and Project Management, RMIT University, Melbourne, \\ Australia
}

\begin{abstract}
This paper proposes to use the nonlinear-autogressive models with exogenous input (NARX) model to predict the hysteretic behaviour of passive control systems. Although existing analytical hysteresis models such as the generalized Bouc-Wen (BW) model and the Bouc-WenBaber-Noori (BWBN) model can be used to model the hysteretic behaviour of passive control systems, the generalized BW model fails to account the pinching or stiffness degradation of hysteretic systems and the BWBN model requires to tune considerable parameters before its application. Therefore, we propose this alternative approach to mimic the hysteresis response of passive control systems. The NARX model is the branch of artificial intelligence which is a promising tool for the forecasting of time series problems. We adopted the NARX model to predict the hysteretic behaviour with experimental results conducted on yielding shear panel device (YSPD) and steel slit damper (SSD), respectively. A good agreement between the experimental results on both YSPD and SSD and the prediction results was achieved. We also

* Corresponding Author: Dr. Eric Wai Ming Lee, E-mail: ericlee@cityu.edu.hk, Fax: (852) 3442 0427, Tel.:

(852) 34422307.
\end{abstract}


combined the NARX model and the general regression neural network (GRNN) as a hybrid model to predict hysteretic behaviour of the SSD of which the damper design was hidden from the model training process. The performance of using the hybrid model to predict the hysteretic behaviour of SSD is reasonably well. Finally, the applicability of the hybrid model has been successfully demonstrated through the optimisation of the geometrical parameters of the SSD. We concluded that the proposed NARX model is capable to predict the hysteretic behaviour of passive control systems.

Keywords: Neural networks; NARX model; GRNN; hysteretic behaviour; passive control system

\section{Introduction}

The past two decades have seen the development of a number of different kinds of structural controls for minimizing the damage of the structures caused by earthquake excitation [1-2]. These structural controls can be broadly divided into passive control systems, active control systems and semi-control systems. Passive control systems, also known as passive energy dissipation systems refer to the systems which do not require any external source of power. It can be achieved by equipping the dampers or designated devices into the structures. In contrast, active control systems refer to the systems which require an external source of power to generate structural control forces. It can be achieved by equipping the real-time processing sensors with force delivery devices into the structures and semi-active control systems refer to the systems which require little power to change certain structural parameters. Unlike active control systems or the semi-active control systems, passive control systems do not require an external source of power, the reliability associated with power supply and computer 
control during the earthquake event is thus eliminated [3]. In addition, by strategically locating the dampers or designated devices in the structure, replacement of the damaged dampers or designated devices can be carried out. Therefore, the passive energy dissipation systems have been considered as an inexpensive and effective way to mitigate the risks caused by earthquake excitation to the structures.

A number of passive energy dissipation devices such as ADAS [4], SSD [3], YSPD [5] and TTD [6] which rely on plastic behavior of metals have been proposed. They are usually incorporated in framed structures by connecting the devices between brace systems and floor beams as shown in Figure 1. The resulting combined lateral stiffness is equivalent to the stiffness of the device and the brace connected in series, in addition to the stiffness of the structure. Hence, the inclusion devices will alter the structural response of the parent frame since the natural frequencies of the structure are modified, and the device will introduce hysteretic damping. Modelling of hysteretic behaviours is important especially in the field of structural engineering as it is impractical to obtain the performances of the dampers solely by experimental approach. It is costly and time consuming especially in optimizing the designs of the dampers. Therefore, this study not only applies the NARX model to mimic the hysteretic response of the passive control systems but also demonstrate how to optimize the geometrical parameters of the passive control systems. The hysteretic behaviour of the passive dampers can be described by different mathematical models [7-11]. The analytical description of hysteretic response was first formulated by Bouc [12] and later generalized by Wen [7] as the generalized Bouc-Wen (BW) model. The generalized BW model proposed by Wen [7] was further extended to the Bouc-Wen-Baber-Noori (BWBN) hysteretic model [8-11] as the BW 
model does not include the properties of pinching and stiffness degradation. The BWBN is thus one of the famous models for simulating the hysteretic systems because it provides smooth hysteretic and also account for pinching and stiffness degradation.

\section{(Proposed location of Figure 1)}

This section introduced the passive energy dissipation systems and the importance of the modelling of hysteretic behaviour. The next section briefly describes the design of the yielding shear panel device (YSPD) employed for the model development in this study. Section 3 explains the reason of using artificial intelligence to predict the hysteretic behaviour of passive control systems. Section 4 introduces the design of the nonlinear-autogressive models with exogenous input (NARX) model, both of the architecture and algorithms of the NARX model will be covered. Section 5 reports the results of the hysteretic behaviour modelled by the NARX model. In section 6, we combine the NARX model and general regression neural network (GRNN) as a hybrid model and use it to predict the steel slit damper (SSD) of which the damper design was hidden from the model training process. We also demonstrated the applicability of the hybrid model through the optimisation of the geometrical parameters of the SSD in section 7. Finally, section 8 provides a conclusion of this paper.

\section{The design of yielding shear panel device (YPSD) and its test}

The design of the YPSD is shown in Figure 2. It is fabricated from a short segment of a square hollow section (SHS) with a steel diaphragm plate welded inside the SHS. The YSPD acts in shear as the parent frame structure undergoes lateral deformation. The input energy is 
dissipated through shear yielding of the diaphragm plate while the relative horizontal displacement between the top and bottom connections of the plate is sufficiently large.

(Proposed location of Figure 2)

Table 1 summaries the details of the test specimens prepared for the test reported in [5]. There are two different SHS sections (i.e., $100 \times 100 \times 4$ and $120 \times 120 \times 5$ ), three diaphragm plate thicknesses (i.e., 2, 3 and $4 \mathrm{~mm}$ ) were used and total 19 tests carried out on the YSPD were reported in [5]. The nomenclature used in the test is $D-t M$ or $D-t C / D-t C S$, where $D$ indicates the size of the SHS section (i.e., 100 or $120 \mathrm{~mm}$ ), $t$ is the thickness of the diaphragm plate (i.e., 2, 3 or $4 \mathrm{~mm}$ ). The letter $M$ and $C$ represent the monotonic test and cyclic test, respectively. The letter $S$ represents a stiffened section.

(Proposed location of Table 1)

Figure 3 shows the experimental setup. The setup was designed and fabricated to ensure the verticality of the applied load. Forced displacement was applied by an MTS $100 \mathrm{kN}$ capacity computer-controlled actuator quasi-statically to the specimen via the L-beam, where a pantograph system was attached to prevent its in-plane rotation. The test specimens were securely fastened by four M16 bolts on each side. The complete test setup rested on a 40-ton reaction frame which was significantly stiffer. A free-run of the setup indicated that effect of friction and gravity was negligible.

(Proposed location of Figure 3) 
Experiment was carried out for each specimen to obtain the transmitted shear force through the damper under the specified forced displacement with 3 consecutive cycles performed at each amplitude (i.e., $0.5,1.0,3.0,5.0,10.0$, and $20.0 \mathrm{~mm}$ ). The time history of the forced displacement and the induced shear force across the dampers were recorded. Readers may refer to [5] for the details of the experiment.

\section{The reason of using Nonlinear-Autoregressive-Exogenous (NARX) model to predict the hysteretic behaviour}

Li et al. [13] successfully applied the BWBN model to simulate the hysteretic behaviour of the yielding shear panel device (YSPD) designed by [5]. The BW model owns the feature of versatility and mathematical tractability. It has widely applied to a variety of engineering problems. The generalized BW model and its extensions like the BWBN model have been used in the modeling and analysis of structural materials [10]. In Li et al. [13] study, Simulink was used to develop the BWBN model of the YSPD. The developed pinching model adopted in [13] provides a reasonable estimation of cumulative energy dissipation (i.e., within 10\%). However, there were up to 16 parameters of the BWBN required to be calibrated to match with the experimental results. It requires a considerable length of time and computational resources to calibrate 16 parameters of the BWBN model. Therefore, this paper proposes an alternative approach to mimic the hysteretic behaviours of the passive energy dissipation systems by the use of artificial intelligence with fewer components to be calibrated.

From historical point of view, moving average, autoregressive moving average and linear parametric were the common approaches for handling the time dependent problems before 
1980s [14]. The algorithms of these approaches are linear and thus they are not applicable to predict the nonlinear time series problems. At the same time, artificial neural networks (ANNs) drew a lot of people attention due to the characteristic of non-linear and non-parametric in nature. ANNs do not require assumptions about the problem under investigation, given that they are data-driven adaptive models and can learn the non-linear behaviour of a system from the historical data of the system. ANNs are suitable for problems whose solutions do not require prior knowledge of the system and they have been proven to be universal function approximators [15], current application of the ANNs can be found in [16-18].

\section{The architecture of NARX model}

Hysteretic systems have memory, the resisting force depends on both instantaneous deformation and past history of the deformation [11]. Therefore, the hysteretic behaviour of the passive control systems shall be suitable to be modelled by the NARX model. The NARX model is one of the recurrent artificial neural network models, which is a powerful class of model for time series prediction. It learns the behaviour of a system in a more effective way than other neural networks (i.e., the learning gradient algorithm is better in NARX) and also converges much faster and generalizes better than other networks [19]. Therefore, the NARX model is not only commonly applied in forecasting the time series cases but also important for the control of the dynamical systems. It has been demonstrated that they are capable to mimic the behaviours of the nonlinear dynamics systems and particularly useful for time series modelling [20-21]. 
The structure of the NARX model is similar to the traditional multi-layered perceptron (MLP) model. Among the various ANN models, the MLP model is one of the most widely used for forecasting due to its simple and flexible nature [22]. Both MLP model and NARX model consist of an input layer, a hidden layer and an output layer but the NARX model feeds the time history of the output signal to the input layer as part of the inputs. The current input signal together with its time history acts as the other part of the inputs to the model. The number of output neurons equals to the number of output variables of the problem to be solved. The number of hidden neurons is required to be determined by the user. Assume $\Gamma$ is the function of the NARX model. It correlates the input time series $\left\{u_{t}\right\}_{t=0}^{T}$ and the output time series $\left\{y_{t}\right\}_{t=0}^{T}$ by the equation (1).

$$
y_{t}=\Gamma\left(y_{t-1}, y_{t-2}, \cdots, y_{t-\tau_{y}}, u_{t}, u_{t-1}, \cdots, u_{t-\tau_{u}}\right)
$$

where $u_{t}$ and $y_{t}$ represent the input and output of the network at time $t$, the function $\Gamma$ is a nonlinear function, $\tau_{y}$ and $\tau_{u}$ are the required lengths of the time histories of the two time series.

(Proposed location of Figure 4)

The general architecture of the NARX model is shown in Figure 4. For this study, the output of the NARX model is the induced shear force across the dampers and the inputs of the NARX model is the time histories of the forced displacement and the induced shear force across the damper. Similar to the MLP model, each neuron has a bias input. The activation functions of the neurons in the input and output layers are linear while that of all hidden neurons is sigmoid 
function. Once the number of hidden neurons is determined, the total number of the weighting factors between each neuron can also be found. For the learning process, the LevenbergMarquardt algorithm is adopted in this study due to the fast convergence properties. The performance index used in NARX model training is the mean squared error (MSE), which is one of the typical performance functions as shown in equation (2).

$$
M S E=\frac{1}{N_{S}} \sum_{i=1}^{N_{S}}\left(t_{i}-y_{i}\right)^{2}
$$

where $t_{i}$ and $y_{i}$ represent the target and the output of the network and $N_{s}$ is the number of training samples.

\section{Prediction of YSPD by the NARX model}

Section 2 detailed the design of the YSPD reported in [5], section 3 and 4 explained the reason of using the NARX model to mimic the hysteretic behaviour and the general architecture of the NARX model, respectively. This section presents the results which predicted by the NARX model. The experimental hysteretic responses of YSPD were reported in [5]. For comparing the results predicted by NARX with Li et al. [13], six of the specimens (i.e., 100-2C, 100-2CS, 100-3C, $100-3 C S, 120-2 C$ and $120-3 C$ ) were used in the current study to evaluate the performance of the trained NARX model. After several trials, it was found that the optimal setting of the required lengths of the time histories of the two time series is 2 (i.e., $\tau_{y}=\tau_{u}=2$ ). Therefore, a two-step-ahead prediction is adopted during the NARX model training process. The architecture of the NARX model adopted in this study to predict the hysteretic response of the YSPD is shown in Figure 5. Only one hidden neuron is used to develop the NARX model throughout this 
study, in which the number of weighting factors to be calibrated for the prediction of hysteretic responses is 8 (i.e., $w_{1}$ to $w_{8}$ ).

\section{(Proposed location of Figure 5)}

The comparison of the experimental hysteretic responses in the cyclic tests of six YSPD and the prediction results by using the NARX model are shown in Figure 6. The crosses in Figure 6 represent the target value which obtained from the experimental study in [5], while the solid lines represent the predicted output which estimated by the NARX model proposed in this study. A positive sign refers to downward induced shear force and displacement in the forcedisplacement hysteresis.

The predicted results of the NARX model show a good agreement with the experimental results. Both of the predicted result and experimental result of Specimen $100-2 \mathrm{C}$ exhibit reasonably stable hysteretic with a slightly pinched hysteretic near zero displacement in the last cycle (i.e., caused by shear buckling). Similar hysteretic behaviour can also be observed for the stiffened specimen $100-2 C S$. Specimens with thicker diaphragm plates (100-3C and 100-3CS) did not show buckling, but exhibit a more pronounced pinched hysteretic loops near the zero displacement due to localized deformation near the bolt connections. Specimen $120-2 \mathrm{C}$ and 120-3C performed satisfactorily with state and large force displacement hysteretic.

(Proposed location of Figure 6)

Li et al. [13] used Simulink to develop the BW and BWBN models to mimic the hysteretic responses of YSPD. There were up to 16 parameters of the BWBN required to be tuned to 
match with the experimental results. In this study, we demonstrated how to use the NARX model with only one hidden neuron to mimic the hysteretic behaivour. Table 2 compares the amount of cumulative energy dissipation predicted by using the BW and BWBN models developed in [13], the experimental results and the NARX models. The area of the hysteresis loops represents the cumulative energy dissipation by the passive energy dissipation systems. The results obtained by Li et al. [13] provide a good estimation of the cumulative energy dissipation, the absolute percentage error of the cumulative energy dissipation between the experimental results and the results predicted by the BWBN models is ranged from $0.57 \%$ to $9.77 \%$ which is superior to the result predicted by the BW models (i.e., ranged from $2.43 \%$ to $52.86 \%)$ as the pinching behaviour was also considered in the BWBN models. On the other hand, the trained NARX models with one hidden neurons provide a very promising estimation of the cumulative energy dissipation, the absolute percentage error of the cumulative energy dissipation between the experimental results and the results predicted by the NARX models is ranged from $0.00 \%$ to $0.75 \%$ for all specimens. It shows a better performance than both $\mathrm{BW}$ and BWBN models with only total 8 numbers of weighting factors to be calibrated. The poor performance of BW and BWBN models may be caused by the over-simplification of the hysteretic behaviour in the differentiation equations of the BW and BWBN models.

(Proposed location of Table 2)

\section{Prediction of SSD by the hybrid model}

Section 5 presented the prediction of the hysteretic behaviour of the YSPD by the NARX model. The prediction results depicted the applicability of the NARX model in hysteretic 
behaviour prediction of passive control systems. In this section, we combine the NARX model and the GRNN model as a hybrid model to predict the mechanical response of a new damper design of which the geometrical parameters of the damper was hidden from the NARX model training. In this study, we adopted the experimental results of SSD from [3] to benchmark the performance of the hybrid model. The design of the experiment of the SSD is introduces as follows.

Figure 7 shows the design of the Steel Slit Damper. It is fabricated from a standard structural I-section with a number of slits introduced the web, forming a vierendeel truss arrangement. The strips are filleted to reduce stress concentrations. Each flange is connected to the main structure by four structural bolts. It is a weld-free design, eliminating heat-affected imperfections associated with welding.

(Proposed location of Figure 7)

Table 3 summaries the dimensions of the six specimens (i.e., SL-01 to SL-06) presented in this study. To eliminate variability in material properties, all specimens were cut from the same structural I-section (152 $\times 152 \times 37$ Universal Column to BS4449). Strip thickness $t$ and material strengths of all specimens were assumed equal. Four $16 \mathrm{~mm}$ diameter holes were drilled on each flange. Two standard test coupons were taken from the web of the section. An average tensile yield stress of $316.5 \mathrm{~N} / \mathrm{mm} 2$ and an average Modulus of Elasticity of 206.1 $\mathrm{kN} / \mathrm{mm} 2$ were obtained by standard tensile tests. Each specimen weighed approximately $2.2 \mathrm{~kg}$. Readers may refer to [3] for the details of the experiment. 


\section{(Proposed location of Table 3)}

Figure 8 shows the domain of the geometrical parameters of the SSD reported in [3]. It can be observed that specimen SL-02 is laid slightly outside the boundary of the domain. In general, the ANN models are developed from the historical data of a system. The ANNs can perform very well for the prediction inside the domain of a system, but not outside the domain of the system. Therefore, we propose to use the hybrid model to predict the hysteretic behaviour of specimen SL-02 from the experimental data of specimens SL-01, SL-03 to SL-06 as the hysteretic behaviour of specimen SL-02 is the nearest specimen to the boundary of the domain.

\section{(Proposed location of Figure 8)}

The hybrid model proposed in this study for mimicking the hysteretic behaviour of the SSD is a combination of the NARX model and the GRNN model. The GRNN was presented by Specht in 1991 [24], which is a memory-based network. It provides estimate of continuous variable and converges to the underlying regression surface even with sparse sample. The GRNN recruits every training sample as a kernel in its model, and thus users do not need to predefine the network structure. It has been successfully applied to a variety of fields such as image processing, non-linear adaptive control and machinery fault diagnosis owing to the simple network structure, fast network training time, powerful regression properties, and ease of implementation [25-27]. 
The GRNN model, in fact, computes the expected conditional mean of a probability distribution in condition with the given input vector. The key step of the GRNN model is to establish the probability distribution density of the joint input and output spaces according to the information of the training samples. Parzen density estimator [28] is employed for the density establishment. Assume $\boldsymbol{X}=\left\{x_{1}, x_{2}, \cdots, x_{n}\right\} \in \mathfrak{R}$ are samples taken from a one dimensional domain with underlying probability density function $\hat{Y}(X)$, the fundamental formulation of the GRNN is deduced as follows.

$$
\begin{gathered}
\hat{Y}(\boldsymbol{X})=\frac{\sum_{i=1}^{N_{S}} Y^{i} e^{\left(-D_{i}^{2} / 2 \sigma^{2}\right)}}{\sum_{i=1}^{N_{S}} e^{\left(-D_{i}^{2} / 2 \sigma^{2}\right)}} \\
D_{i}^{2}=\left(\boldsymbol{X}-\boldsymbol{X}^{i}\right)^{\mathrm{T}}\left(\boldsymbol{X}-\boldsymbol{X}^{i}\right)
\end{gathered}
$$

where $\hat{Y}(\boldsymbol{X})$ is the desired conditional mean associated with the input $\boldsymbol{X}, \sigma$ is the smoothness parameter (i.e., the kernel width of the Gaussian function), $N_{s}$ is the number of training samples, and $D_{i}$ is the Euclidean distance between the training sample, $\boldsymbol{X}$ and the point of prediction $\boldsymbol{X}^{i}$. Different from the traditional MLP or NARX model, once the input of GRNN is given, GRNN is capable to determine the architecture and weights by itself. Therefore, training GRNN is essentially to optimize the smoothness parameter only, to obtain the optimal regression prediction.

The GRNN model is proposed to combine with the NARX model as a hybrid model to predict the hysteretic response of SSD of which the damper design was hidden from the NARX model training. The network structure of the hybrid model is similar to the GRNN model. From 
equation (3) and (4), the hybrid model is constructed as shown in Figure 9. It is with multi inputs and one single output, and consists of 4 layers of neurons, namely input, pattern, summation and output layer.

In this study, the number of the input units in input layer is 2 . These 2 neurons represent the geometrical parameters (i.e., strip length, $l_{O}$ and the strip depth, $d$ ) of the specimen SL-02, respectively. The input layer is connected to the pattern layer and in this layer each neuron presents a training pattern and its output. There are total 5 numbers of neurons in the pattern layer (i.e., specimen SL-01, SL-03 to SL-06 and specimen SL-02 was hidden during the model training process). They calculate the Euclidean distance of the training sample from the neuron's center and apply the Gaussian function with the predefined smoothness parameters (i.e., $\left.e^{\left(-D_{i}^{2} / 2 \sigma^{2}\right)}\right)$. The resulting value is then passed to the neurons in the summation layer. In the summation layer, the numerator summarizes the products of weight values and the resulting values obtained from the pattern layer. In which, the weights $\left(\left\{y_{1}\right\},\left\{y_{3}\right\}\right.$ to $\left.\left\{y_{6}\right\}\right)$ between the pattern layer and the numerator are the time series obtained by the NARX models presented in section 5. For example, the time series $\left\{y_{1}\right\}$ is obtained by the NARX model which developed from the time histories of the displacement and the induced shear force of specimen SL-01 reported in [3]. The denominator also summarizes the products of weight values and the resulting values obtained from the pattern layer. But in this case, the weights on the signals going into the denominator neuron are one. Finally, the output layer divides the accumulated value in the numerator by the value in the denominator and the time series of the induced shear force of specimen SL-02 can be obtained. 
(Proposed location of Figure 9)

(Proposed location of Figure 10)

After several trials, the smoothness parameter $(\sigma)$ was determined to be 50 . Figure 10 shows the hysteretic behaviours of the dampers predicted by the NARX model and GRNN. Table 4 compares the amount of cumulative energy dissipation predicted by using the hybrid model and the experimental results. The amount of cumulative energy dissipation predicted by using NARX models and the hybrid are also presented to benchmark the performance of the hybrid model. The trained NARX models with only one hidden neuron provide very a promising estimation of the cumulative energy dissipation, the absolute percentage error of the cumulative energy dissipation between the experimental results and the results predicted by the NARX models is ranged from $0.00 \%$ to $0.30 \%$ for all the specimens of the SSD. The performance of using the hybrid model to predict the hysteretic behaviour of SSD is reasonably well. The absolute percentage error of the cumulative energy dissipation between the experimental results and the results predicted by the hybrid models of specimen SL-02 is $6.97 \%$.

It concluded that the application of the hybrid model is capable to predict the hysteretic behaviour of a new design of passive control systems.

(Proposed location of Table 4)

\section{Application of the hybrid model to optimize the geometry of the SSD}

This section demonstrates the application of the developed hybrid model with GAs [29] for passive control system design. The advantage of GAs over conventional searching 
algorithms is that GAs can deal with a wide range of areas and are able to find 'acceptable' solutions in a reasonable time. They have also been shown to be superior to conventional optimisation techniques, especially for discontinuous and noisy functions [30].

The passive control system is designed for minimizing the damage of the structures caused by earthquake excitation, the larger the energy dissipated by the damper, the better the performance of the passive control system is. To mitigate the energy generated during the earthquake, the designer is required to determine the geometrical parameters of the passive control damper properly. We propose to use the developed hybrid model with GAs to optimize the geometrical parameters of the SSD reported in [3], such that the energy dissipation can be maximized. Equation 5 is the fitness function to be minimised in the GAs optimisation to achieve this design objective. It can be approximated by using the numerical method as shown in equation 6 . Therefore, the result is converged if the fitness value reaches the minimum.

$$
\begin{aligned}
& \text { fitness value }=\frac{1}{\int_{a}^{b} f(x) d x} \\
& \int_{a}^{b} f(x) d x \approx \frac{\sum_{i=1}^{\frac{b-a}{\Delta x}}\left(f_{i+1}-f_{i}\right)}{2}
\end{aligned}
$$

As explained in section 6, ANN models are developed from the historical data of a system. They perform well for the prediction inside the domain of a system. Therefore, some constraints are listed as follow to provide a bounded condition for the optimization:

1. Lower Boundary is added to ensure the minimum strip depth and length is within the boundary of the domain as reported in [3] (i.e., $b \geqq 14.9 \mathrm{~mm}$ and $\mathrm{l}_{0} \geqq 77.0 \mathrm{~mm}$ ). 
2. Upper Boundary is added to ensure the maximum strip depth and length is within the boundary of the domain as reported in [3] (i.e., $b \leqq 16.9 \mathrm{~mm}$ and $l_{0} \leqq 99.2 \mathrm{~mm}$ ).

The optimization process involve both of the hybrid model and GA model. The main purpose of the hybrid model is to estimate the cumulative energy dissipation of the SSD and the GA model is to search the optimum strip depth and length to minimize the fitness function as shown in equation 5. During the optimization process, the fitness value is converged at approximately 12 generation and the best fitness value is $1.6719 \times 10^{-4}$ (i.e., the cumulative energy dissipation is $5969.47 \mathrm{~kJ}$. The optimised strip depth and length are 16.8853 and 77.0002 , respectively. The process of optimization is shown in Figure 12.

\section{Conclusion}

In this paper, the hysteretic behaviour of the passive control systems in structures is proposed to be modelled by the NARX model. The performance of the prediction is evaluated and the prediction results show a good agreement between the experimental results and the predicted outputs for both YSPD and SSD. The maximum absolute percentage error of the cumulative energy dissipation between the experimental results and the results predicted by the NARX models of the YSPD and SSD are $0.75 \%$ and $0.30 \%$, respectively. It indicates a reliable prediction to the experimental results by using the NARX model. We also presented the hybrid model which is the combination of the NARX model and GRNN model to predict the hysteretic behaviour of SSD of which the damper design was hidden from the NARX model training. The hysteretic behaviour of the SSD can also be successfully modelled by the hybrid model. The absolute percentage error of the cumulative energy dissipation between the experimental results and the result predicted by the hybrid model is $6.97 \%$, which is a reasonably good 
prediction. Finally, the applicability of the hybrid model has been successfully demonstrated through the optimisation of the geometrical parameters of the SSD.

\section{Acknowledgement}

The work described in this paper was fully supported by a grant from the Research Grant Council of the Hong Kong Special Administrative Region [Project no. CityU 116613].

\section{References}

1. Soong TT, Dargush GF. Passive energy dissipation systems in structural engineering. John Wiley \& Sons; 1997.

2. Soong TT, Spencer Jr BF. Supplemental energy dissipation: State-of-the-art and state-ofthe-practice. Eng Struct 2002;24:243-59.

3. Chan RWK, Albermani F. Experimental study of steel slit damper for passive energy dissipation. Eng Struct 2008;30(4):1058-66.

4. Bergman DM, Goel SC. Evolution of cyclic testing of steel plate devices for added damping and stiffiness. Report No. UMCE87-10. Ann Arbor: The University of Michigan; 1987.

5. Chan RWK, Albermani F, Williams MS. Evaluation of yielding shear panel device for passive energy dissipation. Construct Steel Res 2009;65(2):260-8.

6. Benavent-Climent A. A brace-type seismic damper based on yielding the walls of hollow structural sections, Eng Struct 2010;32(4):1113-1132. 
7. Wen YK. Method for random vibration of hysteretic systems. J Eng Mech Div, ASCE 1976;102:249-63.

8. Baber TT, Wen YK. Random vibration of hysteretic degrading systems. J Eng Mech, ASCE 1981;107:1069-89.

9. Baber TT, Noori MN. Modeling general hysteretic behaviour and random vibration application. J Vibr Acoust Stress Reliab Design-Trans, ASME 1986; 108:411-20.

10. Foliente GC, Singh MP, Noori MN. Equivalent linearization of generally pinching hysteretic, degrading systems. Earthq Eng Struct Dyn 1996;25: 611-29.

11. Mostaghel N. Analytical description of pinching degrading hysteretic systems. J Eng Mech 1999;125(2):216-24.

12. Bouc R., Modele Mathematique D’Hysteresis, Acustica, 1967; 24(1): 16-25 (in French).

13. Li Z, Albermani F, Chan RWK, Kitipornchai S Pinching hysteretic response of yielding shear panel device. Eng Struct 2011;33:993-1000.

14. Pollok DSG. A Handbook of Time-Series, Signal Processing and Dynamics. Academic Press, 1999.

15. Hornik K, Stinchcombe M, White H. Multilayer feed forward networks are universal approximators. Neural Networks 1989;2:359-366.

16. Yuen JKK, Lee EWM, Lo SM, Yuen RKK. An Intelligence-Based Optimization Model of Passenger Flow in a Transportation Station. IEEE Trans Intell Transp Syst 2013;14(3):1290-1300. 
17. Yuen RKK, Lee EWM, Lo SM, Yeoh GH. Prediction of temperature and velocity profiles in a single compartment fire by an improved neural network analysis, Fire Saf J $2006 ; 41(6): 478-85$

18. Li DHW, Tang HL, Lee EWM, Muneer T. Classification of CIE standard skies using probabilistic neural networks, Int J Climatol 2010;30(2):305-15.

19. Lin T, Horne BG, Tino P and Giles CL. Learning Long-term dependencies in NARX recurrent neural networks, IEEE Trans Neural Netw 1996;47(6):1329-51.

20. Chen S, Bilings SA and Grant PM. Non-linear system identification using neural networks. Int J Control 1990;51(6):1191-1214.

21. Leontaritis IJ and Bilings SA. Input-output parametric models for non-linear systems: Part IL deterministic non-linear systems. International Journal of Control 1985;41(2): 303-28.

22. Rosenblatt F. Principles of Neurodynamics, Spartan Books, New York; 1962.

23. Ward, Neuroshell 2 Manual, Ward Systems Group Inc, Frederick, MA; 1996.

24. Specht DF. A general regression neural network, IEEE Transaction on Neural Networks $1991 ; 2(6): 568-76$

25. Rzempoluck EJ. Neural network classification of EEG during camouflaged object identification. Int J Med Inform 1997;44:169-75.

26. Schaffner C and Schroder D. An application of general regression neural network to nonlinear adaptive control, EPE 1993;4:219-24. 
27. Hyun BG and Nam K. Faults diagnoses of rotating machines by using neural nets: GRNN and BPN, Proceedings of the 1995 IEEE IECON 21st International Conference on Industrial Electronics, Control, and Instrumentation 1995;2:1456 -61.

28. Parzen E. On estimation of a probability density function and mode, Ann Math Stat $1962 ; 33: 1065-76$.

29. Holland JH. Adaptation in Natural and Artificial Systems, MIT Press; 1975.

30. Beasley D, Bull DR and Martin RR. An overview of genetic algorithms: Part 1, Fundamentals, University Computing 1993;41(2):58-69. 


\section{List of figures:}

Figure 1 Frame-brace-device assembly

Figure 2 Design of the YSPD (a) Elevation (b) Top view (c) Deformed shape

Figure 3 Overview of the experimental setup

Figure 4 General architecture of the NARX model

Figure 5 Architecture of the NARX model adopted in this study

Figure 6 Hysteretic behaviours of the specimens (100-2C to 120-3C) of YSPD predicted by the NARX model

Figure 7 Design of the SSD (a) Elevation (b) Top view

Figure 8 Domain of the geometrical parameters of the SSD

Figure 9 Architecture of the hybrid model (GRNN model with NARX model) 
Figure 10 Hysteretic behaviours of the SSD (SL-02) predicted by the hybrid model

Figure 11 Fitness value versus generation during the optimisation process

\section{List of tables:}

Table 1 Dimensions of the test specimens (unit: $\mathrm{mm}$ ) of YSPD

Table 2 Comparison of hysteretic energy dissipation of YSPD from different models

Table 3 Dimensions of the test specimens (unit: $\mathrm{mm}$ ) of SSD

Table 4 Comparison of hysteretic energy dissipation of SSD from different models 


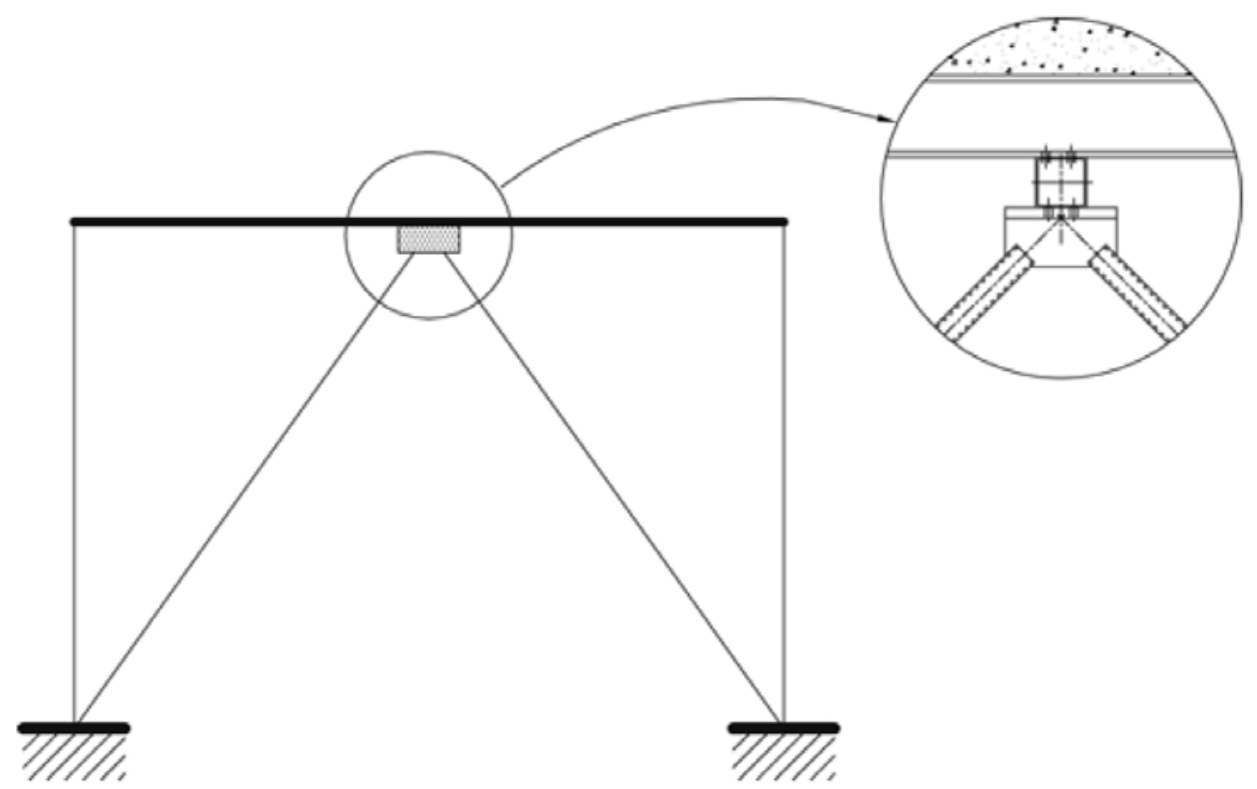

Figure 1 Frame-brace-device assembly 

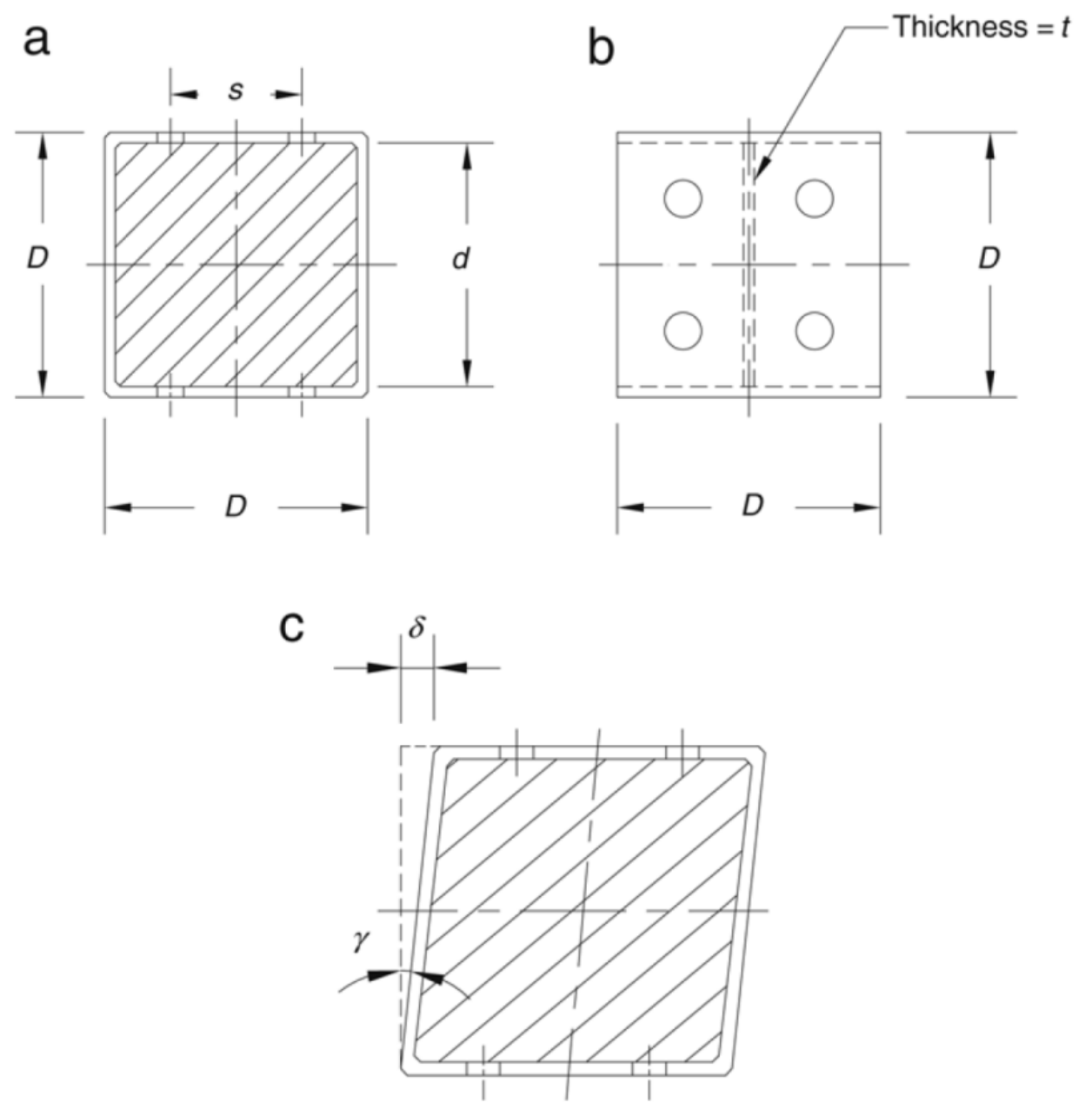

Figure 2 Design of the YSPD (a) Elevation (b) Top view (c) Deformed shape 


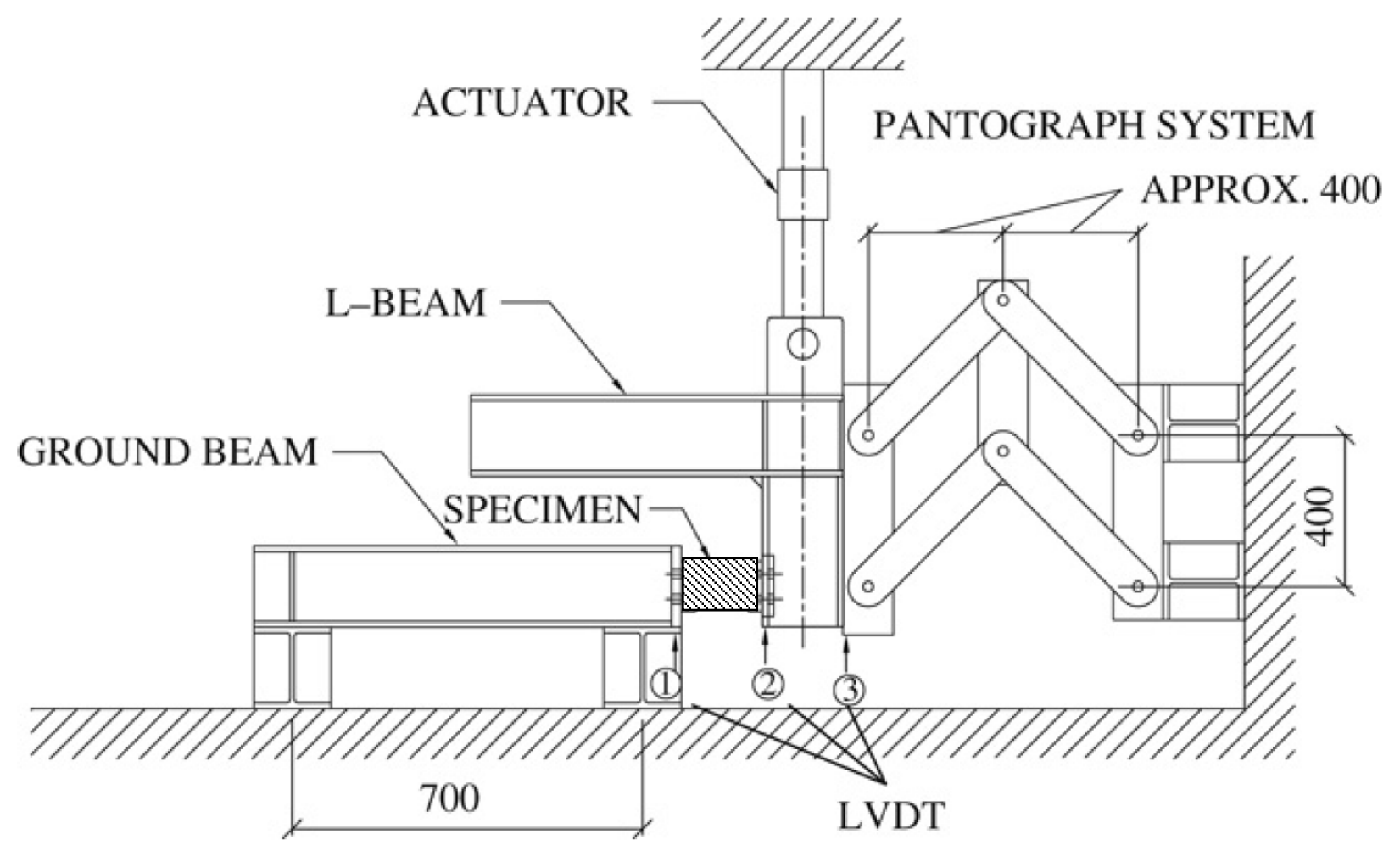

Figure 3 Overview of the experimental setup 


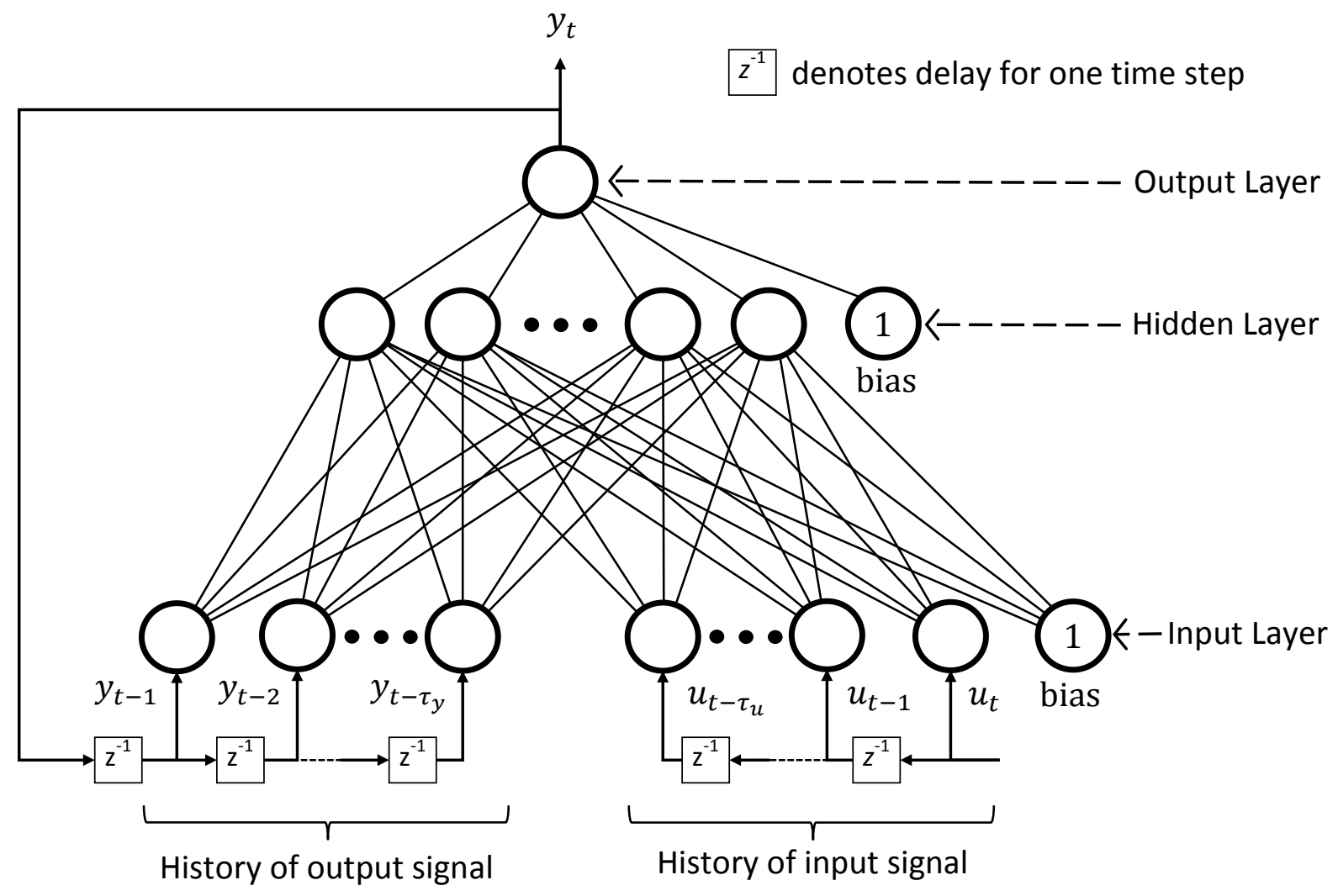

Figure 4 General architecture of the NARX model 


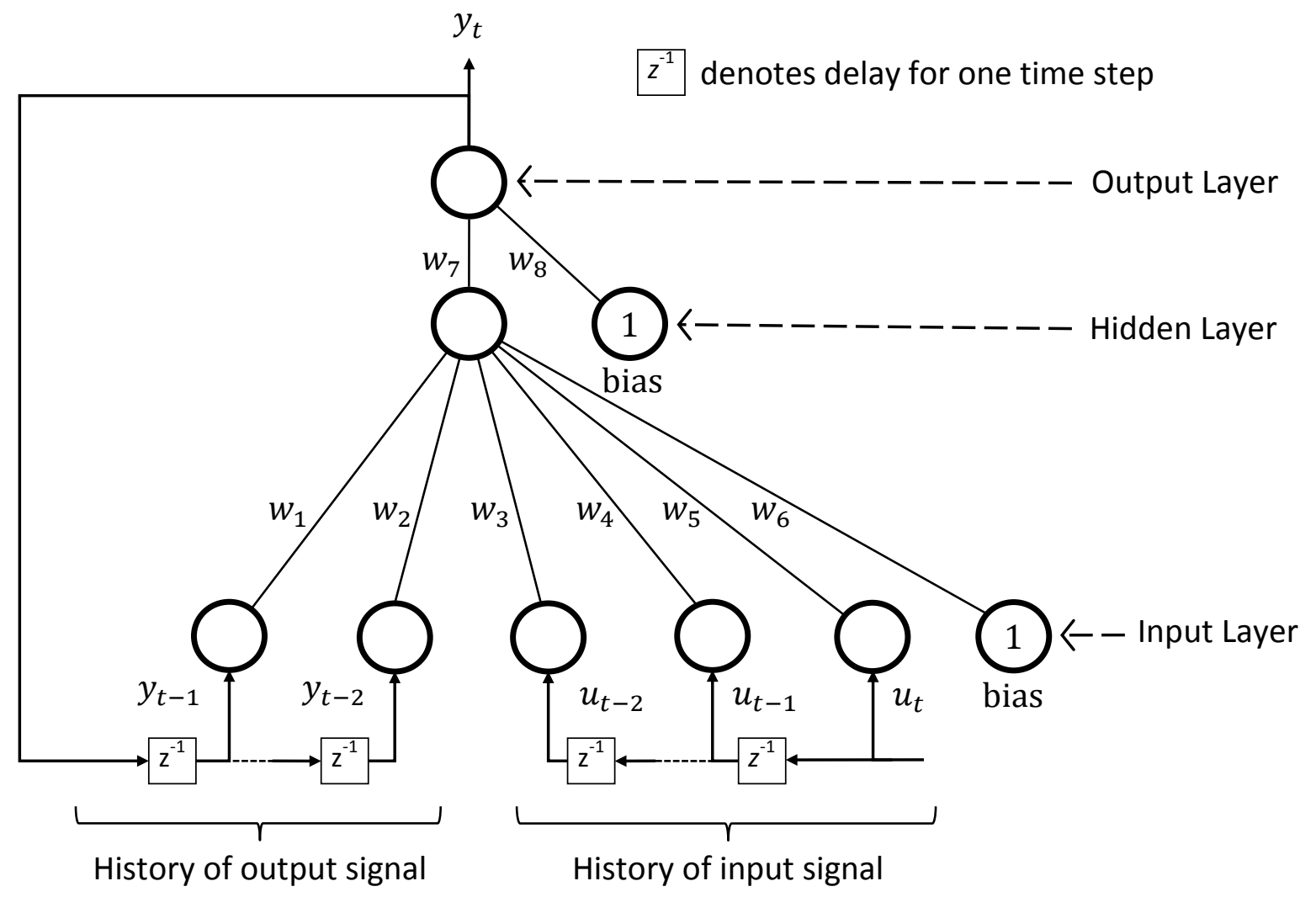

Figure 5 Architecture of the NARX model adopted in this study 

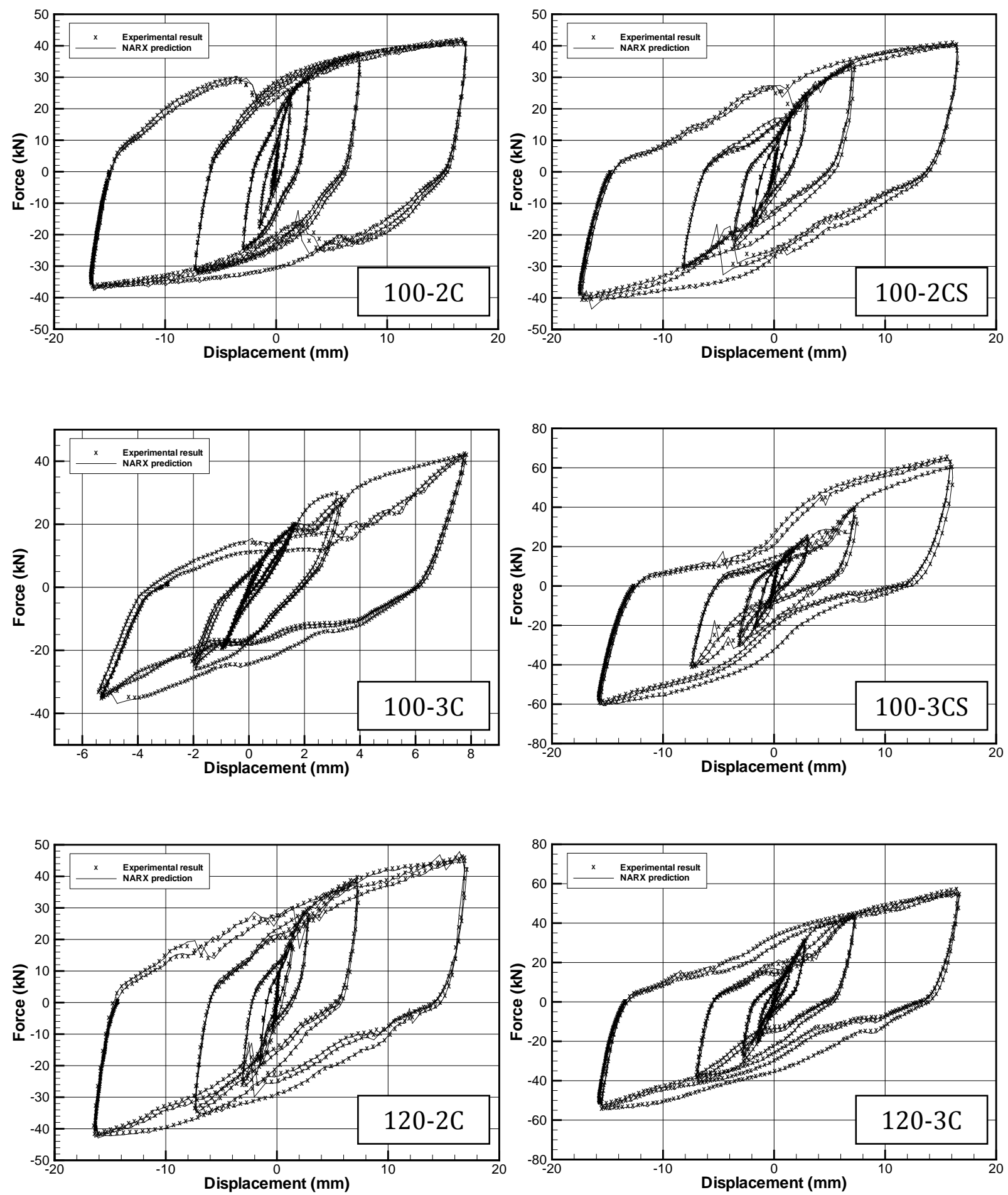

Figure 6 Hysteretic behaviours of the specimens (100-2C to 120-3C) of YSPD predicted by the NARX model 


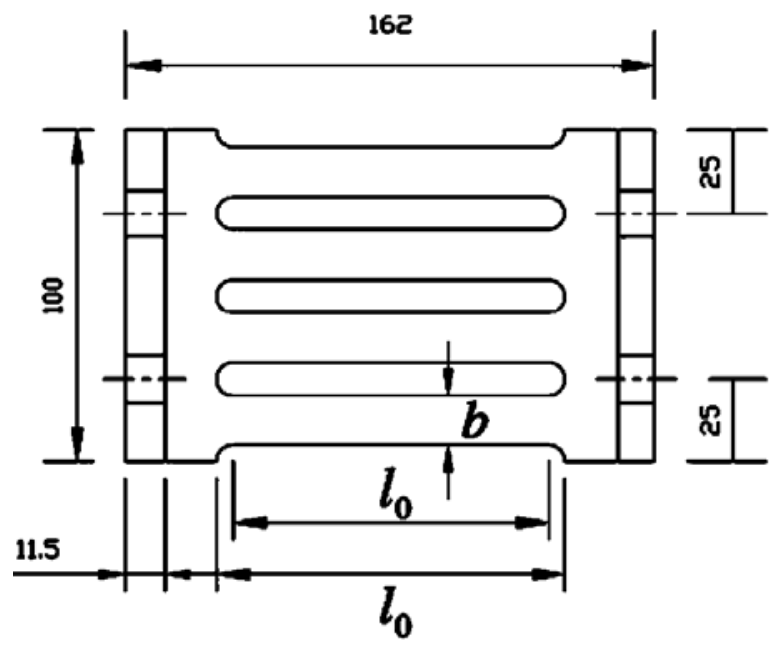

(a)

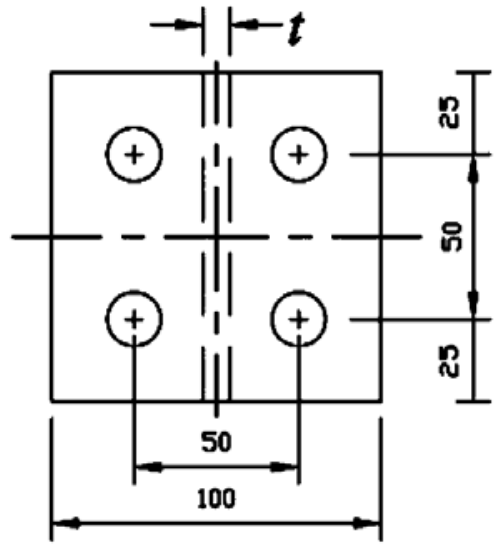

(b)

Figure 7 Design of the SSD (a) Elevation (b) Top view 


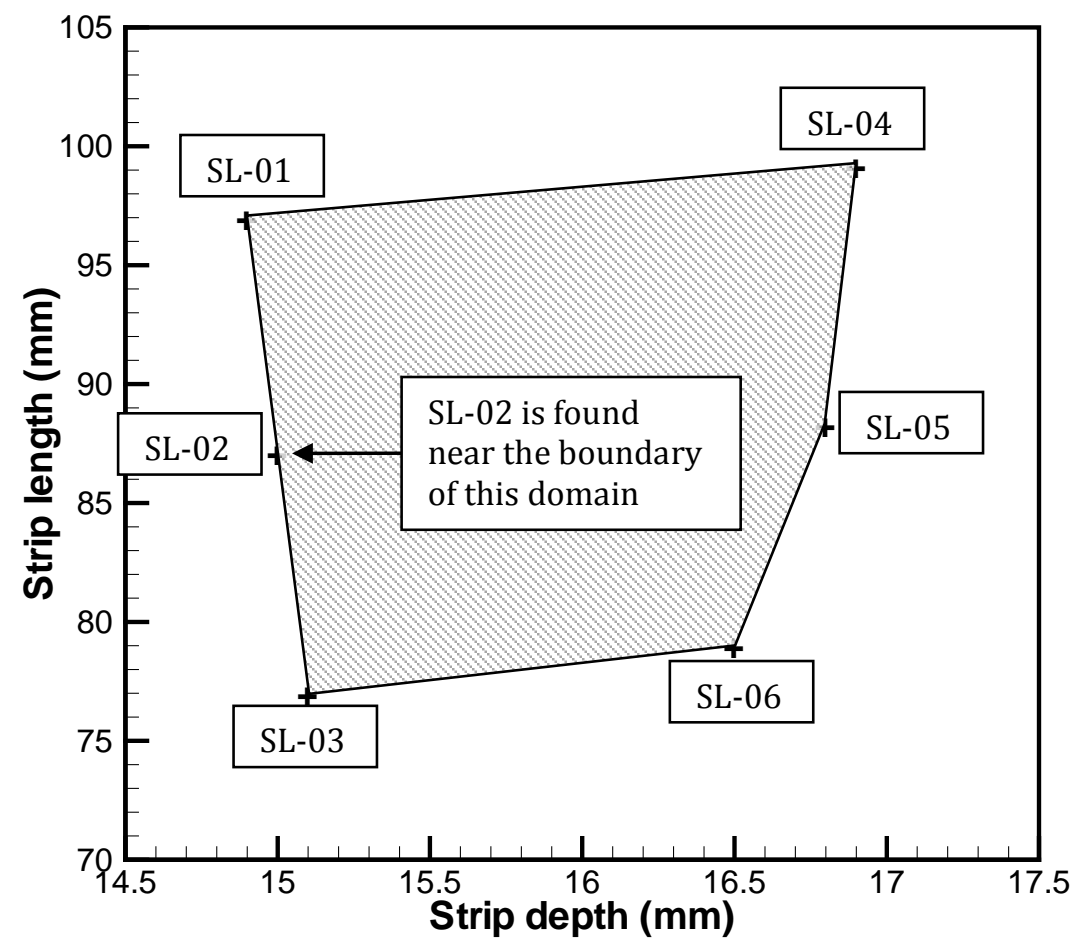

Figure 8 Domain of the geometrical parameters of the SSD 


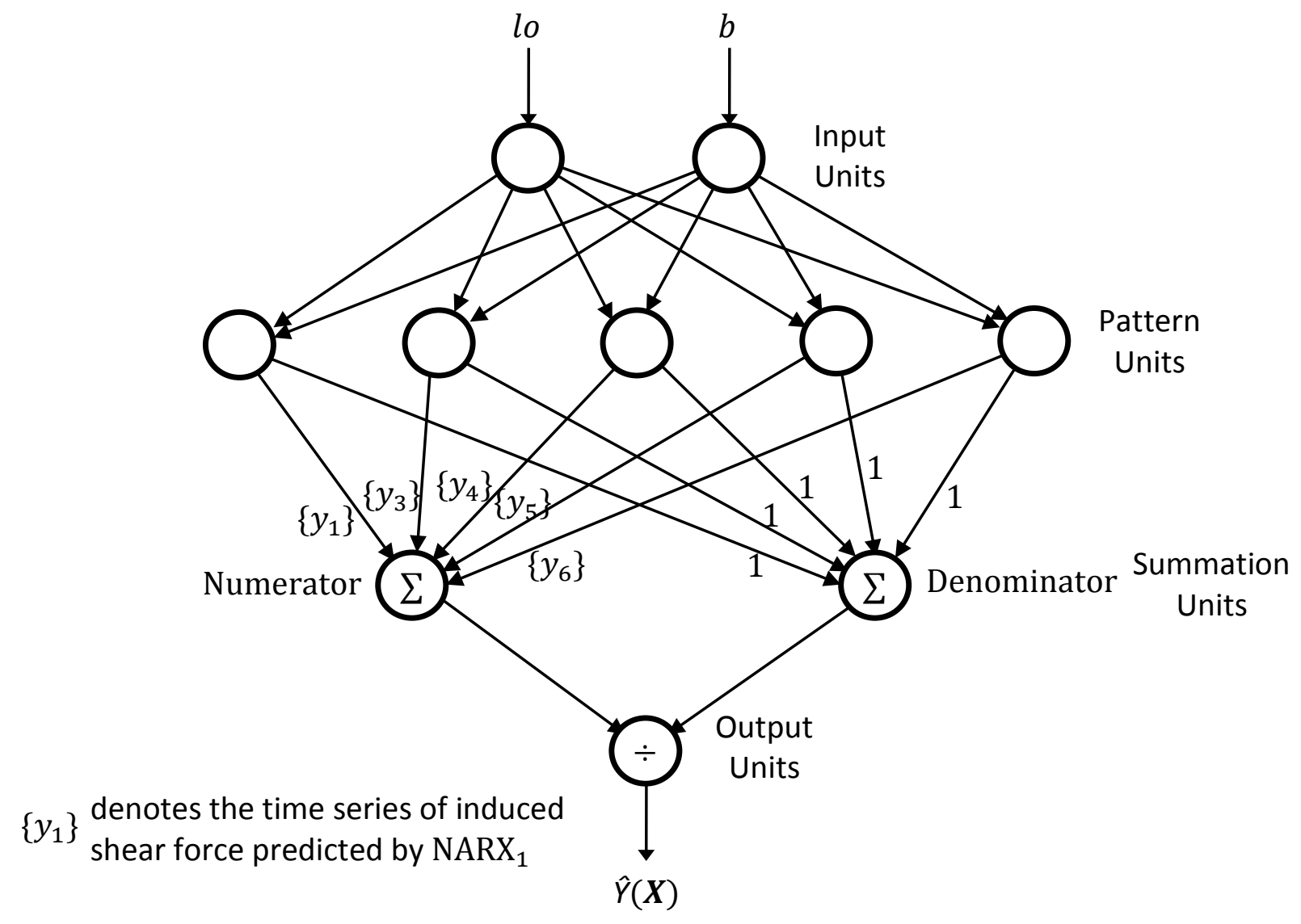

Figure 9 Architecture of the hybrid model (GRNN model with NARX model) 


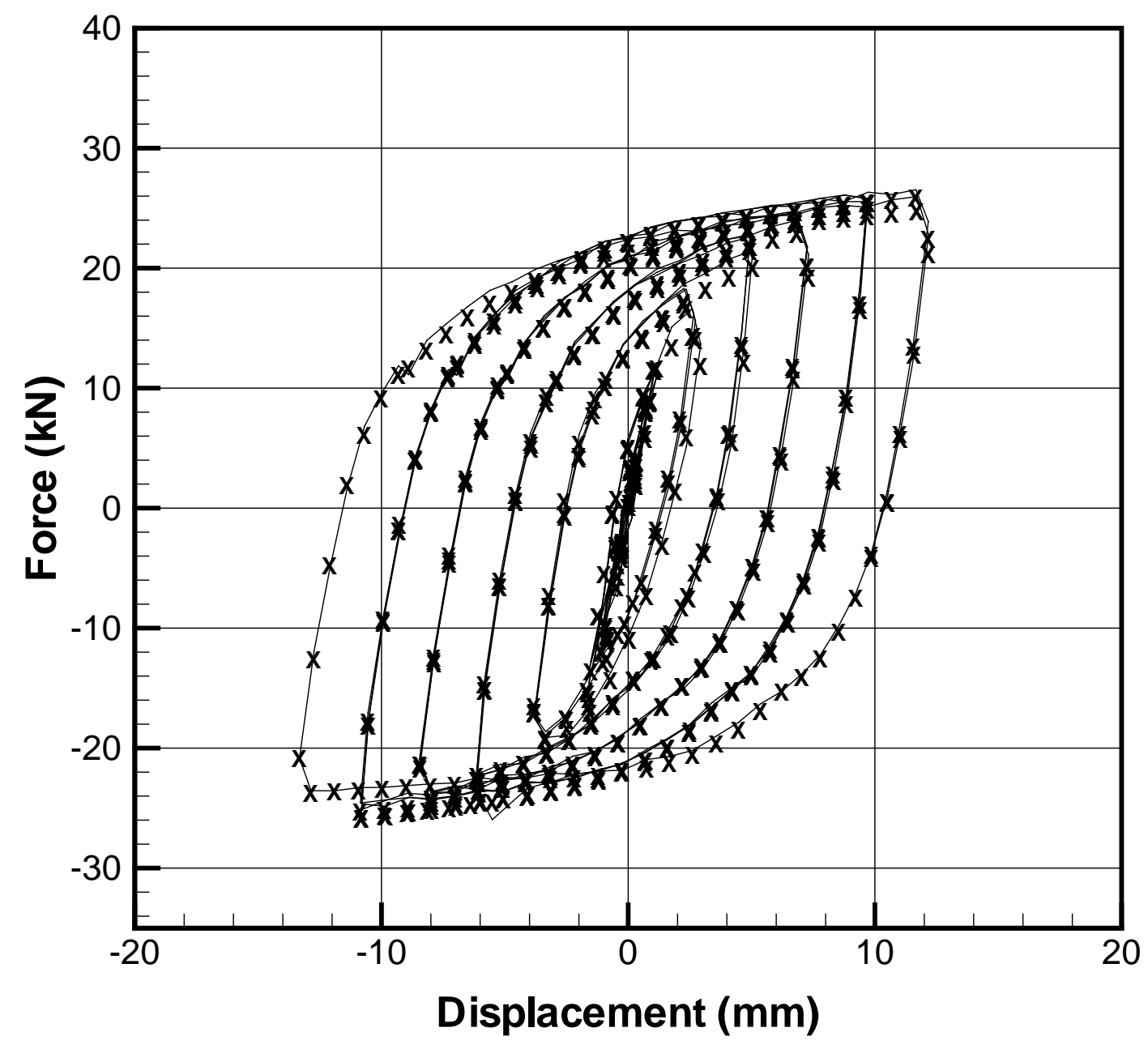

Figure 10 Hysteretic behaviours of the SSD (SL-02) predicted by the hybrid model 


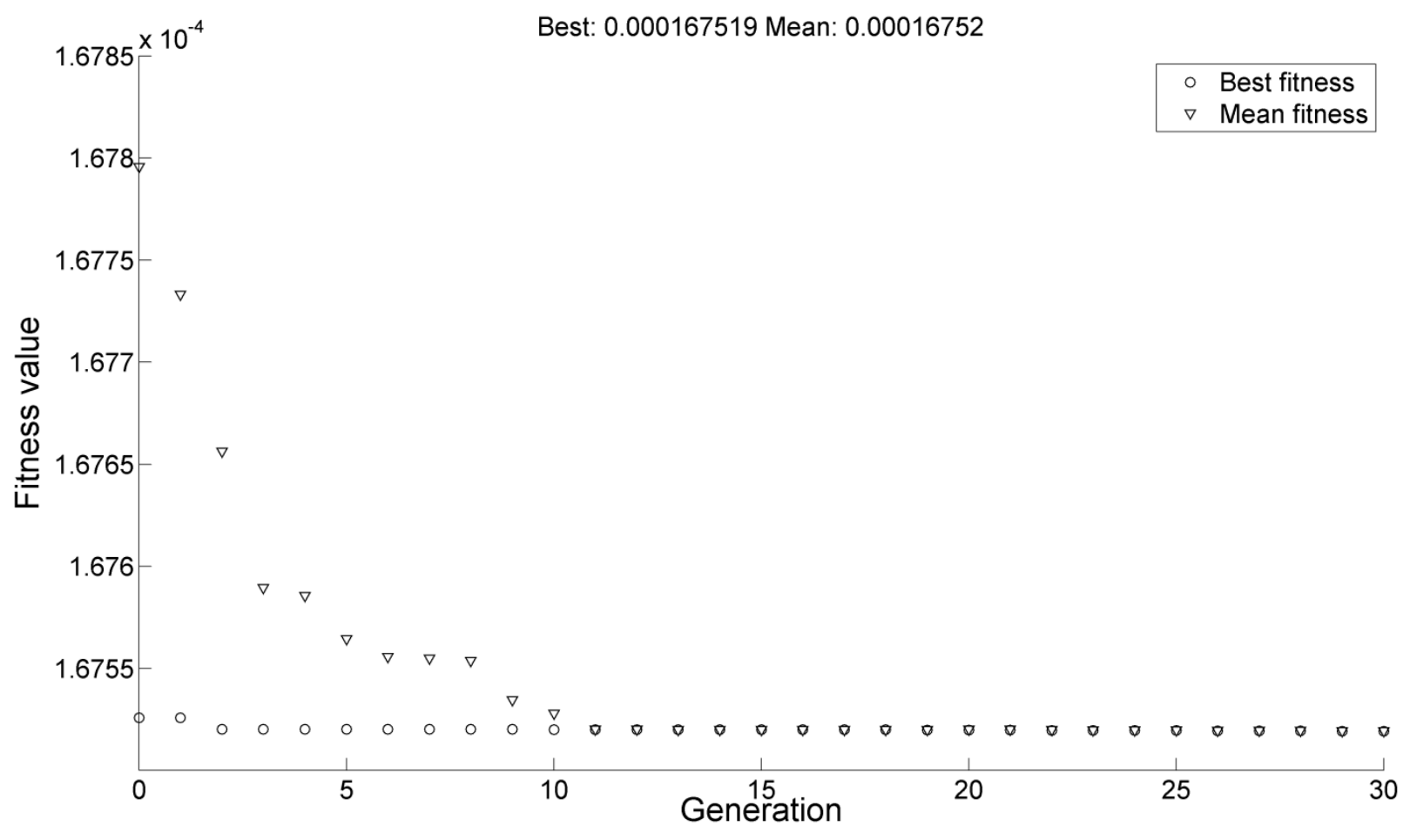

Figure 11 Fitness value versus generation during the optimisation process 
Table 1 Dimensions of the test specimens (unit: $\mathrm{mm}$ ) of YSPD

\begin{tabular}{cccc}
\hline Test number & Specimen & SHS & Test regime \\
\hline 1 & $100-0 \mathrm{M}$ & & \\
2 & $100-2 \mathrm{M}$ & $100 \times 100 \times 4$ & Monotonic \\
3 & $100-3 \mathrm{M}$ & & \\
4 & $100-4 \mathrm{M}$ & & \\
\hline 5 & $100-0 \mathrm{C}$ & & \\
6 & $100-2 \mathrm{C}$ & & \\
7 & $100-3 \mathrm{C}$ & & \\
8 & $100-4 \mathrm{C}$ & $100 \times 100 \times 4$ & \\
9 & $100-2 \mathrm{CS}$ & & \\
10 & $100-3 \mathrm{CS}$ & & \\
11 & $100-4 \mathrm{CS}$ & & \\
\hline 12 & $120-0 \mathrm{M}$ & & \\
13 & $120-2 \mathrm{M}$ & $120 \times 120 \times 5$ & Monotonic \\
14 & $120-3 \mathrm{M}$ & & \\
15 & $120-4 \mathrm{M}$ & & \\
\hline 16 & $120-0 \mathrm{C}$ & & \\
17 & $120-2 \mathrm{C}$ & $120 \times 120 \times 5$ & \\
18 & $120-3 \mathrm{C}$ & & \\
19 & $120-4 \mathrm{C}$ & & \\
\hline
\end{tabular}


Table 2 Comparison of hysteretic energy dissipation of YSPD from different models

\begin{tabular}{ccccc}
\hline \multirow{2}{*}{ Specimen } & \multirow{2}{*}{$\begin{array}{c}\text { Experimental } \\
\text { results }\end{array}$} & \multicolumn{2}{c}{ Energy Dissipation in kJ, (Absolute percentage error) } \\
\cline { 3 - 5 } & & BW model & BWBN model & $\begin{array}{c}\text { NARX with one hidden } \\
\text { neuron }\end{array}$ \\
\hline $100-2 C$ & 6.99 & $7.16(2.43 \%)$ & $6.95(0.57 \%)$ & $6.98(0.14 \%)$ \\
$100-3 C$ & 1.33 & $1.92(44.36 \%)$ & $1.46(9.77 \%)$ & $1.32(0.75 \%)$ \\
$100-2 C S$ & 5.73 & $6.92(20.77 \%)$ & $5.79(1.05 \%)$ & $5.73(0.00 \%)$ \\
$100-3 C S$ & 5.94 & $9.08(52.86 \%)$ & $6.39(7.58 \%)$ & $5.93(0.17 \%)$ \\
$120-2 C$ & 6.23 & $7.28(16.85 \%)$ & $6.45(3.53 \%)$ & $6.21(0.32 \%)$ \\
$120-3 C$ & 6.51 & $7.65(17.51 \%)$ & $7.10(9.06 \%)$ & $6.49(0.31 \%)$ \\
\hline
\end{tabular}


Table 3 Dimensions of the test specimens (unit: $\mathrm{mm}$ ) of SSD

\begin{tabular}{ccccc}
\hline \multirow{2}{*}{ Specimen ID } & \multicolumn{3}{c}{ Measured dimensions } \\
\cline { 2 - 4 } & web thickness, $t$ & strip depth, $b$ & strip length, $l_{O}$ & $b / l_{O}$ \\
\hline SL-1 & 8.0 & 14.9 & 97.0 & 0.155 \\
SL-2 & 8.0 & 15.0 & 87.1 & 0.172 \\
SL-3 & 8.0 & 15.1 & 77.0 & 0.195 \\
SL-4 & 8.0 & 16.9 & 99.2 & 0.172 \\
SL-5 & 8.0 & 16.8 & 88.3 & 0.191 \\
SL-6 & 8.0 & 16.5 & 79.0 & 0.215 \\
\hline
\end{tabular}


Table 4 Comparison of hysteretic energy dissipation of SSD from different models

\begin{tabular}{cccc}
\hline & & \multicolumn{2}{c}{ Energy Dissipation in kJ, (Absolute percentage error) } \\
\cline { 3 - 4 } Specimen & Experimental results & NARX with one hidden neuron & $\begin{array}{c}\text { Hybrid model (NARX } \\
\text { with GRNN models) }\end{array}$ \\
\hline SL-01 & 8.17 & $8.18(0.12 \%)$ & - \\
SL-02 & 6.60 & $6.62(0.30 \%)$ & $7.06(6.97 \%)$ \\
SL-03 & 5.95 & $5.96(0.17 \%)$ & - \\
SL-04 & 8.92 & $8.92(0.00 \%)$ & - \\
SL-05 & 6.79 & $6.79(0.00 \%)$ & - \\
SL-06 & 7.38 & $7.40(0.27 \%)$ & - \\
\hline
\end{tabular}

